\title{
Estrategias de lectura Abana para desarrollar la comprensión lectora de textos argumentativos en estudiantes de educación Superior.
}

\author{
Reading strategies for Abana develop reading comprehension of argumentative \\ texts students in higher education. \\ ${ }^{1}$ Nazario Aguirre B. ", ${ }^{1}$ Edwin Gavidia O. ${ }^{2}$, Máximo Ramírez J. ${ }^{a}$
}

\section{RESUMEN}

El presente trabajo de investigación tuvo como objetivo demostrar los efectos de un programa de estrategias de lectura ABANA para mejorar la comprensión lectora en textos argumentativos en los estudiantes de Educación Superior de la ciudad de Jaén, entendiendo que los estudiantes que inician los estudios universitarios, tienen scrias dificultades en el procesamicnto de la información y en la construcción del significado global del texto.

El problema que da apertura a la investigación surge a raíz de observar que no existen estudios de rigor científico sobre la comprensión lectora a nivel universitario por lo que me motivó a desarrollar la presente investigación; en este sentido, se aplicó un programa de estrategias de lectura, para identificar el nivel literal, inferencial y crítico de la comprensión lectora en textos argumentativos a estudiantes de educación superior de la ciudad de Jaén.

En la presente investigación se trabajó con una muestra de 30 estudiantes de diferentes carreras profesionales y para probar la hipótesis se optó por el diseño pre experimental de tipo de pre test y post test con una sola medición, cuyo instrumento utilizado fue construido y validado por expertos.

Sobre la base del resultado de la contrastación de la hipótesis puedo concluir que la aplicación del programa de estrategias de lectura se obtuvo resultados positivos, tal como se demuestra en la tabla $\mathrm{N}^{\circ} 01$ y gráfica $\mathrm{N}^{\circ} 01$ del Pre Test y Pos Test.

Palabras clave: Estrategias de lectura, comprensión lectora, textos argumentativos.

\begin{abstract}
The present research aimed to demonstrate the effects of a program ABANA reading strategies to improve reading comprehension argumentative texts in Higher Education students in the city of Jaén, meaning that students who start university studies have serious difficulties in processing information and in building the overall meaning of the text.

The problem as it opens to the investigation stems from observing that there are no scientifically rigorous studies on college-level reading comprehension so that motivated me to develop this research, in this sense, we implemented a program of reading strategies to identify the literal, inferential and critical reading comprehension argumentative texts to students of higher education in the city of Jaén.

In this research, we worked with a sample of 30 students from different careers and to test the hypothesis we chose the pre-experimental design of pre test and post test with a single measurement, the instrument was constructed and validated by experts.

Based on the results of the testing of the hypothesis I can conclude that the implementation of the reading strategies were scored positive, as shown in Table No. 01 and No. 01 Graphic Pre Test and Post Test.
\end{abstract}

Keywords: Reading strategies, reading comprehension, argumentative texts.

${ }^{1}$ Universidad Nacional de Jaén. ${ }^{2}$ Universidad Alas Peruanas.

${ }^{a}$ Doctor en educación, ${ }^{b}$ Médico - Cirujano 


\section{INTRODUCCIÓN}

La lectura es un factor primordial en toda clase de aprendizaje personal y enriquecimiento intelectual. Por ello, una buena lectura no debe quedarse en la mera comprensión superficial y literal de lo escrito, se debe tratar de profundizar hasta encontrarse con la propia comprensión e intenciones que transmite el autor.

El problema de investigación surge al observar que los estudiantes de educación superior de las diversas carreras universitarias de la ciudad de Jaén, tienen problemas para comprender textos argumentativos; esto motivó a plantear la siguiente interrogante: ¿Cuáles serán los efectos del programa de estrategias de lectura en el desarrollo de la comprensión lectora de textos argumentativos en estudiantes de educación superior de la ciudad de Jaén?

La presente investigación se sustenta en los postulados de Camasco (2003) y valle Valle (2006), donde estudian la producción de textos argumentativos con características discursivas multimodales en la educación superior.

Desde esta esfera educativa, la lectura constituye uno de los medios de aprendizaje más eficaces porque no solo facilita al estudiante su formación técnica o profesional, sino que el uso sistemático de los diferentes textos fomenta en el lector hábitos de estudio independiente que le servirán para desarrollar habilidades en la expresión oral y escrita, que permiten hacer un uso de la lengua cada vez más correcta, culta y expresiva.

Al respecto Garcia (2004) afirma: "La argumentación ha sido muy descuidada en la educación, evidenciándose que los textos de opinión no aparecen en los manuales escolares, los juicios de valor, y las opiniones morales, siguen siendo expresadas en la escolaridad a través de los relatos, sin que los sujetos se asuman como sujetos discursivos con opiniones explícitamente expresadas".

En este marco, destacando la importancia que tiene la lectura para la formación de buenos estudiantes en educación superior, surge la necesidad de proponer estrategias que contribuyan a formar buenos lectores. Es por ello que el presente estudio, puede servir como punto de partida que lleve a la reflexión y búsqueda de mejores técnicas, estrategias o métodos para mejorar la calidad de la comprensión lectora.

\section{MATERIALES YMÉTODOS}

En el desarrollo del presente estudio de investigación se aplicó un Pre Test y Post Test, mediante el método de la experimentación y se apoyó en la estadística para probar sus resultados.

Es un estudio cuantitativo porque se observaron las variables para poder lograr mayor objetividad en los resultados. Asimismo, podemos afirmar que es una investigación aplicada porque soluciona problemas de la vida práctica de los seres humanos y de la sociedad.

Los principales métodos que se utilizaron en la investigación fueron: observación, análisis, síntesis, inductivo, deductivo.

La observación: proceso de conocimiento por el cual se percibió deliberadamente ciertos rasgos existentes en el objeto de conocimiento.

EI análisis: como procedimiento mental permitió la división mental del todo en sus múltiples relaciones y componentes.

La síntesis: estableció mentalmente la unión entre las partes previamente analizadas, posibilitando descubrir las relaciones esenciales y características generales entre ellas; es decir, la síntesis se produjo sobre la base de los resultados obtenidos previamente en el análisis.

\section{Instrumentos de recolección de datos:}

Documentos, fichas de entrevistas, hojas de encuestas.

\section{Técnicas de procesamiento y análisis:}

Elaboración de tablas y gráficos estadísticos.

\section{RESULTADOS}

Los resultados obtenidos del Pre Test y Post Test, aplicado a 30 estudiantes de educación superior de la ciudad de Jaén, arrojó los resultados siguientes: 
Tabla 1. Comparativa de los resultados obtenidos del pre - test y el post - test

PRE. TEST

POST. T EST

\begin{tabular}{cccccccccccccc}
\hline & LITERAL & \multicolumn{1}{c}{ INFERENCIAL } & CRITICO & LITERAL & INFERENCIAL & CRITICO \\
NIVELES & f & $\%$ & f & $\%$ & f & $\%$ & f & $\%$ & f & $\%$ & f & $\%$ \\
& & & & & & & & & & & & \\
Bajo & 25 & 83,33 & 27 & 90 & 28 & 93,33 & 5 & 16,7 & 2 & 6,7 & 2 & 6,7 \\
Medio & 5 & 16,67 & 3 & 10 & 2 & 6,67 & 10 & 33,3 & 12 & 40,0 & 10 & 33,3 \\
Alto & 0 & 0 & 0 & 0 & 0 & 0 & 15 & 50,0 & 16 & 53,3 & 18 & 60,0 \\
Total & 30 & 100 & 30 & 100 & 30 & 100 & 30 & 100 & 30 & 100 & 30 & 100 \\
& & & & & & & & & & & & \\
\hline
\end{tabular}

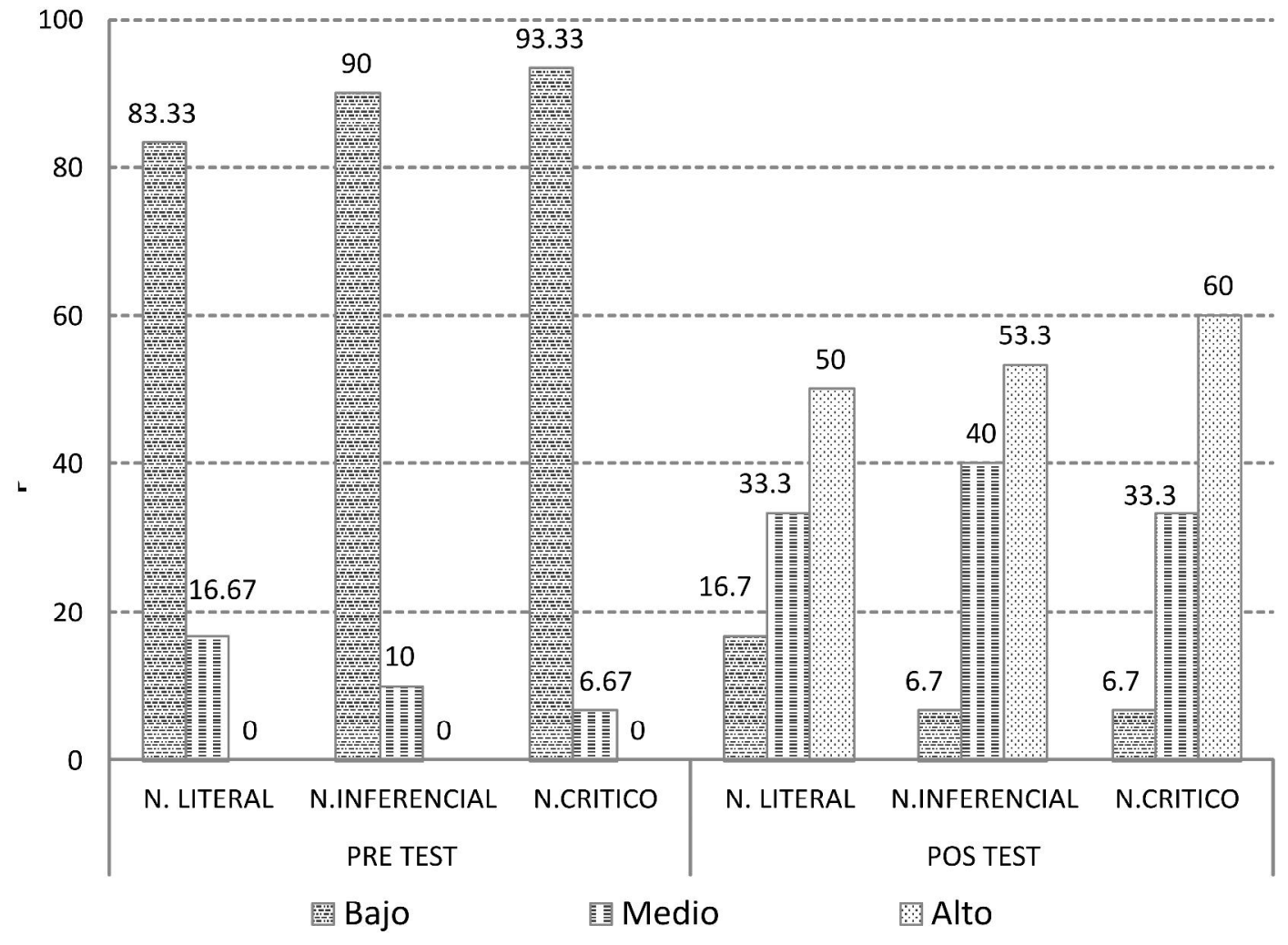


En la figura 01, se puede visualizar los niveles de rendimiento en comprensión lectora de textos argumentativos obtenidos en el pre test, donde los encuestados muestran los siguientes resultados: En el nivel literal, bajo: 83,33\%, medio: $16.67 \%$. En el nivel inferencial: Bajo: $90 \%$, medio: 10\% del total. En el nivel crítico: bajo: $93.33 \%$ y medio: $6.67 \%$.

En cuanto a los niveles en los resultados del post test, los encuestados obtuvieron los siguientes resultados: En el nivel literal, bajo: 16,7\%, medio: $33.3 \%$, alto: $50 \%$. En el nivel inferencial, bajo: $6.7 \%$, medio: $40 \%$, alto: $53.3 \%$. En el nivel crítico: bajo: $6.7 \%$, medio: $33.3 \%$, alto: $60 \%$ del total de los estudiantes. Se puede observar que los valores del post test han aumentado considerablemente en comparación con los resultados obtenidos en el pre test, mostrando un resultado positivo a la aplicación del programa de estrategias de lectura ABANA.

\section{DISCUSIÓN}

Tal como lo señala que el estudio de estrategias de comprensión lectora, lo que se busca es que los docentes que enseñan en educación en su mayoría brinden a sus alumnos estrategias de lectura que le faciliten la comprensión de un texto. En su investigación aplicó algunas estrategias, las cuales permitieron que en su mayoría (70\%) los alumnos fueran capaces de comprender textos, además fueron capaces de crear textos muy originales y creativos. A diferencia, en nuestro trabajo de investigación, se puede notar que existe limitado apoyo por parte de los docentes, pues en este estudio se determinó que el Pre - Test aplicado a 30 estudiantes de educación superior de la ciudad de Jaén, para identificar el nivel de comprensión lectora en textos argumentativos el $83,33 \%$ de los estudiantes se ubicaron en el nivel bajo, cuyos calificativos oscilan entre 0 a 10 puntos; en identificar el nivel literal del conocimiento de la comprensión lectora (Entwidtle, 1988). La comprensión lectora y rendimiento escolar se confirma, que si los estudiantes no son capaces de entender simples textos, mucho más difícil se le hará leer y comprender textos científicos.

En su estudio comprobó las graves defíciencias de los alumnos, pues no hacían uso de estrategias de lectura, en ninguno de los momentos de la lectura. Al contrario de nuestro estudio, según estos resultados se determinó que a los integrantes de este grupo, les falta identificar los tres niveles: nivel literal, inferencial y crítico de la comprensión lectora de los textos argumentativos (Sanchez 2006)

Por otro lado, según Yarba (2007) con su tesis Aplicación de un Programa de Estrategias de Meta comprensión lectora en el desarrollo de habilidades cognitivas en el área de comunicación de los alumnos de cuarto grado de secundaria de la institución educativa Aplicación $\mathrm{N}^{\circ} 10836$ " del distrito de José Leonardo Ortiz de la provincia de Chiclayo, en sus conclusiones establecen que al inicio de la investigación los dos grupos mostraron bajos niveles en habilidades cognitivas $(10,78$ puntos el grupo experimental y 11,06 puntos el grupo control) situación que reflejan deficiencias para comprender datos literales, deducir información y emitir juicios de valor sobre la información textual.

Por consiguiente, en el presente trabajo de investigación, de acuerdo a los resultados obtenidos podemos establecer que la comprensión lectora de nuestro país es deficiente, prueba de ello lo encontramos en los estudiantes encuestados, pues en su mayoría no dedican mucho tiempo a la lectura y por ende tienen dificultades para comprender textos. Pues, para conocer el nivel inferencial de la comprensión lectora de los textos argumentativos, según el Pre Test aplicado a 30 estudiantes determinó que el nivel inferencial del conocimiento de la comprensión lectora se ubica en el nivel bajo. Sin embargo, después de obtener los resultados del Post - Test, aplicado a los 30 estudiantes se determinó que el $16,7 \%$ de los estudiantes se ubicaron en el nivel medio y alto, cuyos calificativos oscilan entre 11 a 20 puntos en identificar el nivel literal del conocimiento de la comprensión lectora.

Según estos resultados se determina que los integrantes de este grupo, identifican el nivel literal, inferencial y crítico de la comprensión lectora de los textos argumentativos después de haber aplicado el programa de estrategias de lectura ABANA; por consiguiente, el promedio alcanzado por los 30 alumnos de este grupo fue de 16,21 2,8 puntos, determinando que el nivel crítico del conocimiento de la comprensión lectora se ubica en el nivel alto. 
Por lo que es necesario, que para poder tener lectores efectivos, crear en los estudiantes hábitos de lectura y sobre todo estrategias que les generen ganas de querer leer un texto, es importante que los estudiantes cuenten con textos con temáticas atractivas y de acuerdo a sus intereses.

\section{CONCLUSIONES}

- La comprensión lectora en textos argumentativos es un tema preocupante en nuestro país, puesto que, los estudiantes presentan un bajo conocimiento de los niveles de la comprensión lectora en los textos argumentativos.

- Las causas del bajo rendimiento sobre comprensión lectora en textos argumentativos son diversas, sin embargo, la más preocupante es que los alumnos, muchas veces, no conocen o simplemente no hacen uso de estrategias de lectura que les ayude a comprender un texto.

- El docente de educación superior, en todas las áreas curriculares, debe orientar a los estudiantes en cada momento la lectura, ya sea en antes, durante y después de la lectura. Además, debe conocer qué estrategias utilizar para cada momento.

- La aplicación del programa de estrategias de lectura ABANA es de vital importancia para desarrollar la comprensión lectora de textos argumentativos en los estudiantes de educación superior.

\section{AGRADECIMIENTOS}

Nuestro especial agradecimiento a la Comisión Organizadora de la Universidad Nacional de Jaén, por las facilidades brindadas durante todo el proceso de la ejecución de la investigación, así como a los profesionales que colaboraron con sus valiosos aportes y sugerencias.

\section{REFERENCIAS BIBLIOGRÁFICAS}

- Álvarez, C. 2006. Didáctica de la Educación Superior. Perú: Editorial FASHSE. Antuña, Roxana 2006.

- Estrategias para la Comprensión Lectora. Disponible en: http://portal.educ.ar/debates /eid/lengua/para-trabajar-clase/estrategias-para-
Camps, Anna 2005. La comprensión lectora, problema de todos. El país digital.

- Carrasco, Alma 2003. La Escuela puede Enseñar Estrategias de Lectura y Promover su Regular Empleo. Revista de Investigación Mexicana. Volumen VIII, num 17:131.

- Entwistle, N. 1988. La comprensión del aprendizaje en el aula. Barcelona. Paidos/ M.E.C.

- García, Antonio 2004. Comunicación y expresión oral y escrita: la dramatización como recurso. Editorial GRAO. Segunda edición. España.

- González, María 2003. La Lectura y la Escritura como Estrategia para el Aprendizaje Autónomo. http:/www.psicocentro.com/cgibin/articulo_s.as p?texto $=\operatorname{art} 23002$

- Ortiz, Marielsa 2006. Propuesta didáctica para la enseñanza de la lectura y la escritura en la educación básica. Edita y Distribuye: Fe y Alegría. Movimiento de Educación Popular e Integral.

- Smith, Frank 1990. Para darle sentido a la lectura. Editorial Visor. España.

- Sánchez, Hugo 1996. Metodología y diseños en la investigación cientifica. Segunda Edición. Págs.

- Valle, Maritza 2006. El problema de la lectura en el Perú.

- Yabar, Luis 2007. Punto de vista del plan lector como estrategia plan lector: lineamiento $y$ formulación.

\section{Correspondencia}

Nazario Aguirre Baique.

Villanueva Pinillos No 720 - Jaén

nabunj@hotmail.com 